\title{
Genetic Programming and Evolvable Machines: Five years of Reviews
}

The journal, and in particular the resource reviews have been running for more than five years. Now is a good time to revisit our original goals [1], compare them with what the journal has achieved and make new plans. Section 2 onwards, updates the statistics on the state of the genetic programming, evolvable hardware and evolvable machines literature and electronic resources.

\section{Five Years of Resource Reviews}

Excluding special issues, every issue of GP/EM has contained at least one review, making a total of 26 . It was intended from the start that these would cover, not just books, but "resources" in the wider sense, particularly, web pages, on-line resources, packages and products.

We have reviewed 18 books, 5 edited collections, and two conference/workshop proceedings. We have also published an article on Internet based resources, one on software (albeit covering three packages) and reviewed one product. Topics have included not only genetic programming (14) and evolvable hardware (4) but also 8 other evolvable machine topics: evolutionary programming, evolutionary robotics, particle swarm optimisation, quantum computing, data mining, evolutionary design, as well as genetic algorithms (2). Articles have been written in the USA (8), England (7), Spain (2), Canada (2), Ireland, New Zealand, Brazil, Australia, Germany, Singapore and France. We have published all the reviews promised in [1].

In future reviews, I would like to further expand the trend to reviewing exciting high quality current books from adjacent evolutionary topics as well as from genetic programming, and increase the number of reviews covering aspects of evolvable hardware. We should also continue to seek reviews covering evolvable machines and other non-book resources. However, a journal like GP/EM, is not appropriate for immediate dissemination, therefore we will not review any more conferences or workshops, since many of their results are quickly updated. Although four commercial packages have been considered, only one has led to a review. While three academic platforms have been reviewed. We have also published one review of the web. Future reviews could cover online teaching aids (applets, slides, demos).

I would like to include other media, such as DVDs. However, with the notable exception of Koza and the increasing publication of conference proceedings on CDROM, the GP/EM field has made little use of non-web based electronic media.

We have received a little feedback from readers. Generally this has been positive. People have expressed a preference for short reviews rather than longer. The journal web site http://www.kluweronline.com/issn/1389-2576 often includes one or two reviews amongst its list of most viewed GP/EM articles. In view of this, and increasing pressure from page limits, in future most reviews will be two pages. 


\section{GP/EM Books and Other Resources}

Genetic programming and evolvable machines remains principally scientific in character, with most visible work being carried out by scientists who publish their results. Thus we can get a picture of the field from its scientific output. However any picture is bound to be incomplete, in particular our approach will unfortunately miss applications of GP or EM that have not been published, e.g. because they are too sensitive to release.

\section{Books}

More than 20 GP books have been published in English. Those reviewed in Genetic Programming and Evolvable Machines are shown with *.

- John Koza's 3 seminal tomes each of which is accompanied by a videotape or DVD Genetic Programming, II, III [2] ${ }^{\star}$ and $I V[3]^{\star}$.

- Three edited collections in MIT press' Advances in Genetic Programming series []$^{\star}$.

- Genetic Programming - An Introduction [5] $]^{\star}$. A comprehensive summary and GP's first text book.

- Jacob's Illustrating Evolutionary Computation with Mathematica [6] ${ }^{\star}$.

- Langdon and Poli's Foundations of Genetic Programming book on GP theory []$^{\star}$.

- John Koza's genetic programming book series, which now includes seven volumes. 1. Langdon's Data Structures and Genetic Programming [8 ${ }^{\star}$. 2. Ryan's Automatic Re-engineering of Software Using Genetic Programming [9] ${ }^{\star}$. 3. Wong and Leung's Data Mining Using Grammar Based Genetic Programming and Applications [10] ${ }^{\star}$. 4. O'Neill and Ryan's Grammatical Evolution: Evolutionary Automatic Programming in a Arbitrary Language [11]*. 5. Koza et al.'s GP IV (above). 6. The first Ann Arbor GP workshop Genetic Programming Theory and Practise. (The 2004 workshop will also be published in this series.) 7. Spector's Automatic Quantum Computer Programming.

- There are also a number of other books on advanced technical material, often derived mostly from $\mathrm{PhD}$ thesis projects. Among them are Nordin's Evolutionary Program Induction of Binary Machine Code and its Applications, Dracopoulos' Evolutionary Learning Algorithms for Neural Adaptive Control, Babovic's Emergence, Evolution, Intelligence; Hydroinformatics - A study of Distributed and Decentralised Computing using Intelligent Agents, Frey's Virtual Ecosystems - Evolutionary and Genetic Programming from the perspective of modern means of Ecosystem Modelling (in German) and Kleinau's Application of Genetic Programming to Finance and Operations Management. 
- There are some foreign language GP books. E.g. Iba's Genetic Programming (in Japanese) and Nordin and Johanna's Humanoid (in Swedish)

- While not a GP book, Forsyth's Machine Learning applications in Expert Systems and Information Retrieval [12] contains chapters describing his Beagle system, perhaps the earliest example of artificial evolution of program trees. Here the trees are used to classify data.

In Evolvable Machines there are also a number of books:

- Tomassini and Sanchez's Towards Evolvable Hardware: The Evolutionary Engineering Approach. This is based on the 1995 Lausanne workshop on evolvable hardware but also includes introductory material.

- Thompson's prize winning Hardware Evolution. Automatic Design of Electronic Circuits in Reconfigurable Hardware by Artificial Evolution $\left[13{ }^{\star}\right.$.

- Mange and Tomassini's Bio-Inspired Computing Machines: Towards Novel Computational Architectures [14] .

- Sipper's Evolution of Parallel Cellular Machines: The Cellular Programming Approach (1997) and The Coming Age of Bio-Inspired Computing (2002).

- Sekanina's Evolvable Components: From Theory to Hardware Implementations.

- Zebulum et al.'s Evolutionary Electronics: Automatic Design of Electronic Circuits and Systems by Genetic Algorithms [15] .

Books in related areas include:

- Nolfi and Floreano's The Biology, Intelligence, and Technology of Self-Organising Machines on evolutionary robotics [16]*.

- Dorigo and Colombetti's Robot Shaping: An Experiment in Behaviour Engineering.

- Freitas' Data Mining and Knowledge Discovery with Evolutionary Algorithms $[17]^{\star}$.

- Kumar and Bentley's On Growth, Form and Computers.

- Bentley's Evolutionary Design by Computers [18 $]^{\star}$.

\section{Proceedings}

The EuroGP series of conferences (and accompanying Evo Workshops) has continued to be an annual event for genetic programming. All the papers, even from the first event in 1998, are available online. In 1999, when the journal started, both GECCO (a joint conference between GP and ICGA) and CEC conference 
series started. GECCO and CEC continue to publish many GP and evolvable hardware papers. In the evolvable machines arena, ICES continues to meet every two years, while the NASA/DoD workshop on evolvable hardware has become an annual fixture. Recently the Asia-Pacific workshops on Genetic Programming (ASPGP) started.

Other evolutionary computation conferences also accept GP/EM papers or even have GP/EM tracks. Examples include PPSN, WSC, ACDM, Artificial Evolution (France), A Life, ECML, ICANNGA, FOGA, SAB, PKDD, IJCAI, IJCNN, ICML, AAAI, ECAI, ICSB, Operations Research, ICPR, EWLR, PSB, SEAL, FLAIRS, BNAIC (Holland/Belgium), CIRAS, NWGA (Scandinavia), SIGKDD and AROB (Japan).

\section{Publication Summary}

Regarding GP, many of the statistics presented here are derived from the GPbibliography ${ }^{1}$. The bibliography is available from the Internet in a variety of formats and locations. Unfortunately, no similar effort has been undertaken for the literature on evolvable hardware. As a result statistics can only be presented for GP.

As of November 2004 there were 2940 GP entries in the GP bibliography (excluding late breaking papers, unpublished, miscellaneous, master thesis and undergraduate students and some short posters). Figure 1 shows the number of each entry according to when they where published and by type. Naturally most papers were published in conference proceedings. Initially there was an exponential rise, with the number of publications doubling every year from 1988 to 1996. This has been followed by a rapid linear increase since 1997. To some extent the apparent reduction in growth may be an artifact of the data collection process. The effort available to maintain the bibliography has not increased exponentially. A lot of effort was devoted to ensuring the GP bibliography was as complete as possible up to 1996 (corresponding to the publication of Advances in Genetic Programming volume 2 which contains an annotated bibliography of GP [19]). Since then there have been GP publications which have escaped recording in the bibliography.

Figure 2 plots the number of people active in GP (i.e. according to the GP bibliography they published in a given year). The total number of people who have published GP related papers is about 1795 .

\subsection{Usage of the GP Bibliography}

The GP bibliography can be searched via the collection of computer science bibliographies ${ }^{1}$. The Artificial Intelligence collection of bibliographies is the $3^{\text {rd }}$ largest computer science collection. Within the AI collection, the GP bibliography is the $7^{\text {th }}$ largest. In the year 2003, the computer science collection of bibliographies serviced nearly 4 million search requests, i.e. nearly 10,000 requests per day. In the last 12 months (since July 2003), there has been approximately 4,770 page views of the GP bibliography home page at the universities of Birmingham and Nottingham. 

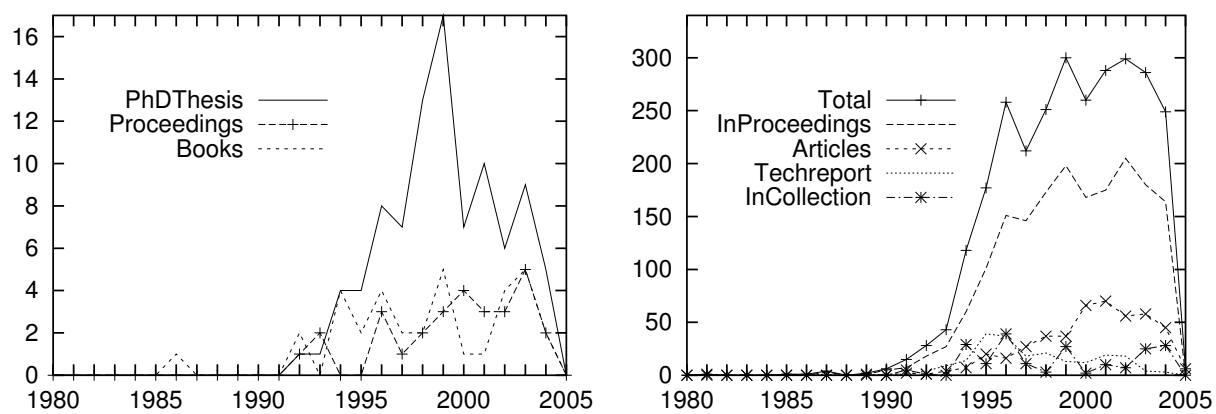

Figure 1. Number of genetic programming entries in the gp-bibliography, according to when they where published and by type. We have excluded late breaking papers, unpublished, misc, masters thesis, undergraduate students and some short posters. Note 20 fold change in vertical scale between left and right.

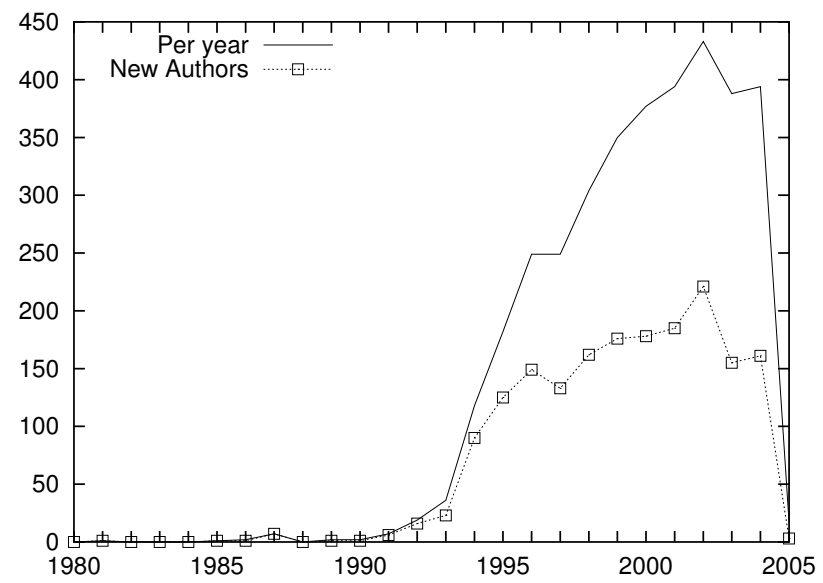

Figure 2. Approximate number of authors and co-authors of genetic programming entries in the gp-bibliography (1980 - November 2004). We exclude late breaking papers, unpublished, misc, masters thesis, undergraduate students and some short posters. The lower curves plots the number of authors who had not previously published in GP.

If we include down loading the bibliography and related files this represents about 550 per month.

Online electronic versions of papers can be directly linked to the bibliography. There are 1477 such links to GP publications $(\approx 90 \%$ of these are valid). In other words half GP papers are immediately available via the Internet. Approximately $45 \%$ of all conference proceedings, $54 \%$ of journal articles, $32 \%$ of papers in collections, $82 \%$ of technical reports and $63 \%$ of $\mathrm{PhD}$ theses are online (linking to HTML, postscript or PDF files). 


\section{Internet}

The GP community has always made extensive use of the Internet. For example with the early establishment (in 1992) of the GP mailing list. (Now to be found at genetic_programming@yahoogroups.com). However the GP ftp archive and FAQ have not been maintained. (Old papers and code can be found at ftp: //cs.ucl.ac.uk/genetic/ftp.io.com/). The FAQ and GP notebook have been supplanted by the use of individual home pages. This means newer material is often scattered across the world wide web. However, while by no means as comprehensive at the biological literature, Google, CiteSeer, the arXiv e-Print archive and various computer science bibliographies, do give rapid access to a large numbers of GP/EM papers. The evolvable machines community has never felt the need for centralised ftp servers and perhaps wisely jumped directly to using distributed world wide web pages. However a summary www page can be found at http://www.cogs.susx.ac.uk/users/adrianth/EHW_groups.html.

Back in November 1997 Altavista listed more than 76 different sites directly related to GP (excluding personal home pages). By September 1999 there were approximately 310 personal home pages written by people interested in GP or evolvable machines. Today (Nov 2004) this has risen to about 870 (see http://www.cs. ucl.ac.uk/staff/W.Langdon/homepages.html for GP pages).

\section{Products}

The number of products making GP or Evolvable Hardware available to ordinary users or incorporating GP is still small. Less than a handful are known. These include: AIM Learning Systems' Discipulus [20] $]^{\star}$, Aber Genomic's Gmax-Bio and Gmax-DM, Cap Gemini's OMEGA, and Hanke and Hörner's Genetic Programming Control.

\section{Freeware}

There is a wealth of freely available software $[21]^{\star}$. For example Source Forge alone lists 21 GP projects. This may be why most researchers continue to use either home grown software or public domain software provided by other researchers. For example Zongker's lilgp ${ }^{2}[22]^{\star}$ has proved popular, with updated versions available both from Michigan State University and Sean Luke ${ }^{3}$. Also in C is Andre's $D G P C^{4}$. There are many $\mathrm{C}++$ implementations, e.g. EODEV $V^{5}$ and Gagne's open Beagle $^{6}$ (not to be confused with Forsyth's Pascal Beagle). While Java implementations include Sean Luke's $E C J^{7}$ and $D R E A M^{8}$. Other implementations, include Koza's Lisp code ${ }^{9}$ and implementations in $C, C++$, SmallTalk, Mathematica, Pro$\log$, Matlab $^{10}$ (e.g. GPLAB ${ }^{11}$ ) and Perl ${ }^{12}$.

There are also several non standard GP systems available. These include Spector's PushGP ${ }^{13}$, O'Niell and Ryan's Grammatical Evolution ${ }^{14}$ and Olsson's ADATE ${ }^{15}$. 


\section{Summary}

I have described the GP/EM literature, our community's heavy use of the Internet, the books and products we have reviewed and indicated plans for future reviews.

\section{Acknowledgements}

I would like to thank Julian F. Miller, John Koza, Maarten Keijzer and Stefano Cagnoni.

\section{Notes}

1. The GP bibliography http://www.cs.bham.ac.uk/^ wbl/biblio//was started from John Koza's bibliography (published in Genetic Programming II 23 ). Over the years I have been greatly assisted in the maintenance and expansion of the GP bibliography by the subscribers to the genetic-programming electronic mailing list.

Direct ftp://ftp.cs.bham.ac.uk/pub/authors/W.B.Langdon/biblio/gp-bibliography.bib

Via the collection of computer science bibliographies

http://liinwww.ira.uka.de/bibliography/Ai/genetic.programming.html etc.

To add entries http://www.cs.ucl.ac.uk/cgi-bin/staff/W.Langdon/WBT.cgi

2. lilgp (in C) http://garage.cse.msu.edu/software/software-index.html

3. Sean Luke's patched lilgp http://www.cs.umd.edu/users/seanl/gp/patched-gp/

4. Dave Andre's DGPC (C) http://www.genetic-programming.com/dgpc.tar.gz

5. EO C++ templates-based evolutionary computation library http://eodev.sourceforge.net//

6. Gagne's open Beagle C++http://www.gel.ulaval.ca/ beagle/

7. Sean Luke's ECJ (Java) http://cs.gmu.edu/ ${ }^{\sim e c l a b / p r o j e c t s / e c j / ~}$

8. DREAM, Distributed Resource Evolutionary Algorithm Machine (Java) http://dr-ea-m.sourceforge.net/

9. Koza's original Lisp http://www.genetic-programming.org/gplittlelisp.html

10. Older implementations in $\mathrm{C}, \mathrm{C}++$, Matlab, SmallTalk, Mathematica and Prolog ftp://cs.ucl.ac.uk/genetic/ftp.io.com/

11. Silva's GPLAB GP tool box for MATLAB http://gplab.sourceforge.net/

12. Bob MacCallum's PerlGP http://perlgp.org/

13. PushGP (Lisp) http://hampshire.edu/lspector/push.html

14. Grammatical Evolution http://www.grammatical-evolution.org

15. ADATE www-ia.hiof.no/ rolando/ 


\section{References}

1. William B. Langdon. Genetic programming and evolvable machines: Books and other resources. Genetic Programming and Evolvable Machines, 1(1/2):165-169, April 2000.

2. Conor Ryan. Book review: Genetic programming 3: Darwinian invention and problem solving. Genetic Programming and Evolvable Machines, 1(4), October 2000.

3. Cyril Fonlupt. Book review: Genetic programming IV. Genetic Programming and Evolvable Machines. Forthcoming.

4. Bruce Edmonds. A review of the "Advances in Genetic Programming" series (volumes 1, 2 and 3). Genetic Programming and Evolvable Machines, 1(3):289-296, July 2000.

5. William Punch. Book review: Genetic programming-an introduction: On the automatic evolution of computer programs and its applications. Genetic Programming and Evolvable Machines, 2(2):193-195, June 2001.

6. Marc Ebner. Book review: Illustrating evolutionary computation with mathematica. Genetic Programming and Evolvable Machines, 4(3):291-294, September 2003.

7. Richard J. Povinelli. Book review: Foundations of genetic programming. Genetic Programming and Evolvable Machines, 5(3):319-320, September 2004.

8. Lance D. Chambers. Book review: Genetic programming and data structures: Genetic programming+data structures=automatic programming. Genetic Programming and Evolvable Machines, 2(3):301-303, September 2001.

9. Kiarash Mahdavi and Mark Harman. Book review: Automatic re-engineering of software using genetic programming. Genetic Programming and Evolvable Machines, 3(2):219-221, June 2002.

10. Alex A. Freitas. Book review: Data mining using grammar-based genetic programming and applications. Genetic Programming and Evolvable Machines, 2(2):197-199, June 2001.

11. Manuel Alfonseca and Alfonso Ortega. Book review: Grammatical Evolution: Evolutionary automatic programming in an arbitrary language. Genetic Programming and Evolvable Machines, 2004. Prepublication Date: 03/23/2004.

12. Richard Forsyth and Roy Rada. Machine Learning applications in Expert Systems and Information Retrieval. Ellis Horwood series in artificial intelligence. Chichester, UK, 1986.

13. Timothy G. W. Gordon. Book review: Hardware evolution: automatic design of electronic circuits in reconfigurable hardware by artificial evolution. Genetic Programming and Evolvable Machines, 2(4):409-411, December 2001.

14. Garrison W. Greenwood. Book review: Bio-Inspired computing machines: Towards novel computational architectures. Genetic Programming and Evolvable Machines, 2(1):75-78, March 2001.

15. Susan Stepney. Book review: Evolutionary Electronics: Automatic design of electronic circuits and systems by genetic algorithms. Genetic Programming and Evolvable Machines, 2004. Prepublication Date: 03/23/2004.

16. Takashi Gomi. Book review: Evolutionary robotics: the biology, intelligence, and technology of self-organizing machines. Genetic Programming and Evolvable Machines, 4(1):95-98, March 2003.

17. David J. Hand. Book review: Data mining and knowledge discovery with evolutionary programs. Genetic Programming and Evolvable Machines, 4(3):287-289, September 2003.

18. Peter A. Whigham. Book review: Evolutionary design by computers. Genetic Programming and Evolvable Machines, 2(1):79-84, March 2001.

19. William B. Langdon. A bibliography for genetic programming. In P. J. Angeline and K. E. Kinnear, Jr., editors, Advances in Genetic Programming 2, pages 507-532. MIT Press, 1996.

20. James A. Foster. Review: Discipulus: A commercial genetic programming system. Genetic Programming and Evolvable Machines, 2(2):201-203, June 2001.

21. Brad Dolin and J. J. Merelo. Resource review: A web-based tour of genetic programming. Genetic Programming and Evolvable Machines, 3(3):311-313, September 2002.

22. G. C. Wilson, A. McIntyre, and M. I. Heywood. Resource review: Three open source systems for evolving programs-lilgp, ECJ and grammatical evolution. Genetic Programming and Evolvable Machines, 5(1):103-105, March 2004.

23. John R. Koza. Genetic Programming II: Automatic Discovery of Reusable Programs. MIT Press, 1994 